\title{
A morphometric and histological study of placental malaria shows significant changes to villous architecture in both Plasmodium falciparum and Plasmodium vivax infection
}

\author{
Sethawud Chaikitgosiyakul', Marcus J Rijken ${ }^{2,3}$, Atis Muehlenbachs ${ }^{4}$, Sue J Lee ${ }^{2,5}$, Urai Chaisri', \\ Parnpen Viriyavejakul ${ }^{1}$, Gareth D Turner ${ }^{1,2,5^{*}}$, Emsri Pongponratn ${ }^{1}$, Francois Nosten ${ }^{2,3,5}$ and Rose McGready ${ }^{2,3,5}$
}

\begin{abstract}
Background: Malaria in pregnancy remains a major health problem. Placental malaria infection may cause pathophysiological changes in pregnancy and result in morphological changes to placental villi. Quantitative histomorphological image analysis of placental biopsies was performed to compare placental villous architecture between active or treated placental malaria cases and controls.

Methods: A total of 67 placentas were studied from three clinical groups: control patients who did not have malaria $(n=27)$, active $(n=14)$ and treated $(n=26)$ malaria cases, including both Plasmodium falciparum and Plasmodium vivax infections. Image analysis of histological placental sections was performed using ImageJ software to measure the number and size (area) of terminal villi, perimeter measurement per villus and total perimeter per unit area, and number of capillaries per villus (vascularity). Histological features of placental malaria were scored and these results were correlated with malaria status and clinical outcomes.

Results: Villous size correlated with vascularity $(p<0.0001)$ but was inversely correlated with observed villi per unit area, $(p=0.0001)$. Significantly greater villous area and vascularity was observed in UK controls. Indices of histological malaria infection were significantly greater in active versus treated malaria cases. Active placental malaria cases showed significantly smaller villous area $(p<0.0084)$, vascularity $(p<0.0139)$ and perimeter $(p<0.0006)$ than treated malaria cases or controls, but significantly more villi per unit area $(p<0.0001)$. Villous size in treated malaria cases was significantly larger than active placental malaria cases $(p<0.001)$ and similar to controls. There was a significant relationship between villous number and anaemia at the time of infection $(p<0.0034)$, but not placental weight, birth weight or gestational age at delivery. No differences were found between histology or villous morphology comparing infections with $P$. falciparum or $P$. vivax.

Conclusions: These results imply that villous size, perimeter and vascularity are acutely decreased during active placental malaria, decreasing the surface area available for gas exchange per villus. However the increased number of villi per unit area offsets this change and persists after treatment. Histopathological and villous architectural changes may be reversed by early detection and appropriate anti-malarial treatment.
\end{abstract}

Keywords: Malaria, Placenta, Pregnancy, Pathophysiology, Morphometry, Image analysis, Villi

\footnotetext{
* Correspondence: gareth@tropmedres.ac

'Department of Tropical Pathology, Faculty of Tropical Medicine, Mahidol

University, 420/6 Rajvithi Road, Bangkok 10400, Thailand

${ }^{2}$ Mahidol-Oxford Research Unit, Faculty of Tropical Medicine, Mahidol

University, 420/6 Rajvithi Road, Bangkok 10400, Thailand

Full list of author information is available at the end of the article
} 


\section{Background}

Malaria infection during pregnancy is a major public health problem in tropical and subtropical regions throughout the world, with substantial risks for the mother, her foetus and subsequently the neonate [1]. Malaria in pregnancy, defined as malaria infection in the placenta or maternal blood at any time during gestation, is associated with a number of materno-foetal complications, such as intra-uterine growth restriction (IUGR), anaemia and pregnancy loss [2-5]. The structure of the placental villous tree changes during normal placental development due to major physiological changes occurring in the placenta during maturation, reflected in changes to the morphology of the stem, intermediate or terminal villi and blood vessels within them [6-8]. Placental malaria, namely the presence of parasitized erythrocytes in the placenta, is inferred to occur during maternal malaria infection, but is only histologically confirmed if parasites are present on histological examination of the placenta following delivery.

Placental malaria infection causes pathological changes such as inflammation, intervillous fibrin deposition and infarction, that can persist until delivery and affect the function of the materno-placental unit, contributing to adverse birth outcomes and increased perinatal mortality and morbidity [9]. However the effect of malaria infection on villous architecture has not been studied in depth. Previous studies have used histomorphometric image analysis to examine placental villous architecture in other diseases causing IUGR, including pre-eclampsia $(\mathrm{PE})$ and diabetes [10-16]. There are a number of physiological factors that can affect villous architecture, as measured on image analysis. Both the age and parity of the mother have been shown to affect villous numbers and vascularity. For instance, the volume density of capillaries in terminal villi was shown to be significantly lower in older women in comparison to the placenta in younger mothers [7]. In idiopathic IUGR, the number of capillaries in the terminal villi and the villous area was significantly decreased compared with controls appropriate for gestational age group $[10,11]$. In PE, the villous area and vascularity were significantly smaller than controls [13]. This study aimed to examine villous architecture and vascularity in placental malaria, relate these to clinical outcomes and the patterns of histopathological changes that have been characterized previously in malaria. It also sought to determine whether any such changes differed between infections with either Plasmodium falciparum and Plasmodium vivax, or were ameliorated by effective anti-malarial treatment before delivery.

\section{Methods}

\section{Ethical approvals}

Ethical permission for collection of placental biopsies in the specific studies was granted with each separate study
[17-21]. Use of these data and specimens in this morphometric study was approved by the Ethics Committee of Faculty of Tropical Medicine, Mahidol University (TMEC 12-027).

\section{Patient selection and clinical groups}

In this study a total of 67 placental biopsies were examined, from cases that were divided into three main clinical groups - non-malaria controls $(n=27)$, active placental malaria cases $(\mathrm{n}=14)$ and treated malaria cases $(\mathrm{n}=26)$.

The active or treated malaria-infected patients $(n=40)$ included $\mathrm{n}=26$ who had only $P$. falciparum infection, $\mathrm{n}=5$ with mono-infection by $P$. vivax and $\mathrm{n}=9$ with consecutive or mixed P. falciparum/P.vivax infections. Of this latter group, $\mathrm{n}=7$ had multiple consecutive infections, including at least one episode each with either P. falciparum and $P$. vivax, and two of whom had proven mixed infections with both organisms simultaneously. Details of the malaria status of the cases are shown in Figure 1.

Placental biopsies were collected between 2004-2011, from the Department of Tropical Pathology, Faculty of Tropical Medicine and three previously published studies investigating malaria in pregnancy conducted at Shoklo Malaria Research Unit (SMRU) field research station at Mae Sot, Thailand. These were studies of the histopathology of placental malaria, used to identify cases with acute infection at delivery with both $P$. falciparum and $P$. vivax [17], an observational ultrasound in pregnancy study (UPS) [18,19] and the artemether-lumefantrine treatment of malaria study (PCA) [20,21]. Women in these studies included ethnic Karen refugees and migrants from an area of approximately $120 \mathrm{~km}$ around the border between Thailand and Myanmar, surrounding the town of Mae Sot and the Maela refugee camp. They attended weekly antenatal clinics for malaria screening and all parasitaemic episodes were treated according to WHO guidelines. As a comparator group for villous morphology in a geographically distinct group of normal control non-malaria cases, a series of normal placental biopsies from the UK were also examined.

The cases included:

(i) Control normal placentas from UK women delivering at The John Radcliffe Hospital, Oxford, UK $(n=9)$, with no fever complicating pregnancy or delivery.

(ii) Control placentas from Karen women delivering at SMRU, who had no evidence of malaria infection by active screening by microscopy throughout pregnancy and negative histology at delivery ( $\mathrm{n}=18$, from UPS study).

(iii) Active placental malaria cases $(n=14)$, where malaria was diagnosed by microscopy and 


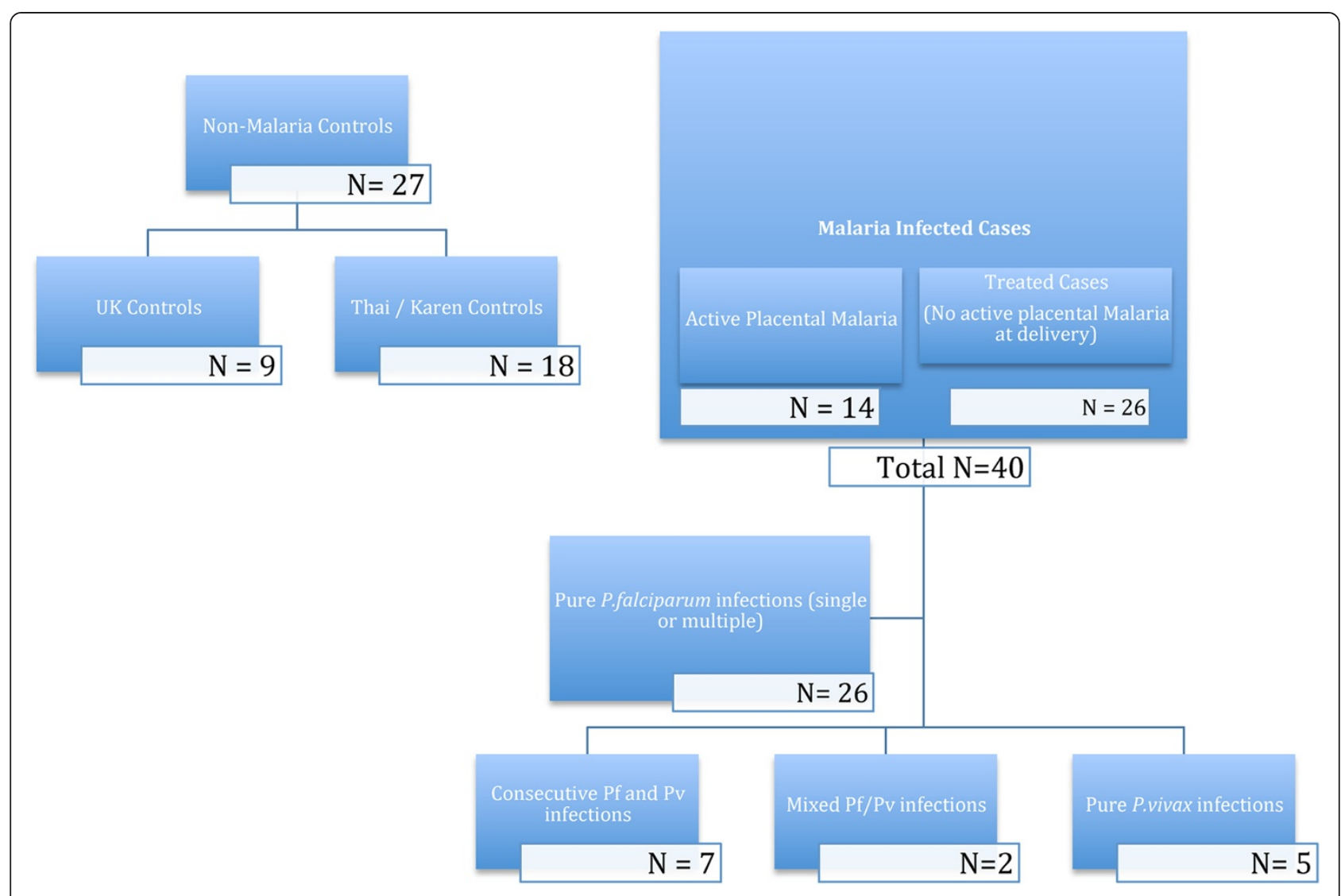

Figure 1 Flow chart showing the type and number of the cases in active and treated malaria and control groups.

subsequent blood films in the mother, and placenta biopsies confirmed acute placental malaria infection at the time of delivery (from histopathology and UPS studies).

(iv) Cases with known single or multiple malaria infections treated during pregnancy at various time points prior to delivery $(\mathrm{n}=12$, from UPS study, $\mathrm{n}=3$ of whom had active placental malaria at delivery and are therefore already included in group iii above).

(v) Single-treated malaria infection in early, middle or late pregnancy (from PCA) but not at delivery $(n=17)$, selected randomly from patients where mono-infection was proven and subsequent screening microscopy until delivery was negative [19]. 'Early' infection $(n=6)$ included women who had a clinical episode of malaria treated at least five months prior to delivery; 'middle' $(n=6)$ included those with malaria infection two to four months before delivery and 'late' infection $(n=5)$ those with treated malaria infection within one month before delivery.

Clinical outcome data on the patients included the gestational age at delivery (EGA), the placental weight at delivery (in grams), the birth weight of the baby (in grams), and the presence of maternal anaemia at the time of infection. This was measured using standard haematocrit (HCT) measurement, and stratified according to the criteria used at SMRU for defining maternal anaemia 1: HCT $>30 \%$ - normal, 2: HCT 25-30\% - mild anaemia and $\mathrm{HCT}<25 \%$ - severe anaemia. EGA was measured for all Mae Sot patients by dating ultrasound prior to 24 weeks, Dubowitz estimation of gestational age and the last menstrual period. Because malaria in this study group was diagnosed actively and prospectively, and treated when found regardless of symptoms, the possibility of asymptomatic placental infection at delivery was minimal. Each case was defined at delivery as being malaria negative on the basis of smear microscopy on cord blood, maternal blood and placental smears. Summary details of the clinical groups are shown in Table 1.

\section{Specimen preparation}

Placental biopsy samples were taken by trained personnel according to established protocols, taking a full thickness biopsy including maternal and fetal surfaces, half way between the placental edge and the cord insertion site. Specimens were fixed in 10\% neutral buffered formalin (UPS, and histopathology study and UK control patients) 
Table 1 Descriptive data summarising the different clinical groups by malaria status

\begin{tabular}{|c|c|c|c|c|c|c|c|c|c|c|c|}
\hline Clinical group & Diagnosis & $\begin{array}{l}\text { Maternal age } \\
\text { at delivery } \\
\text { (years, } \\
\text { mean +/- SD) }\end{array}$ & $\begin{array}{l}\text { Estimated } \\
\text { gestational age } \\
\text { at delivery (weeks, } \\
\text { mean +/- SD) }\end{array}$ & $\begin{array}{l}\text { Median } \\
\text { parity } \\
\text { (range) }\end{array}$ & $\begin{array}{l}\text { Median } \\
\text { number of } \\
\text { malaria } \\
\text { episodes (range) }\end{array}$ & $\begin{array}{l}\text { No. of cases } \\
\text { with pure Pf } \\
N=26 \\
\text { (\% cases) }\end{array}$ & $\begin{array}{l}\text { No. of cases } \\
\text { with pure } \\
\mathrm{Pv} \mathrm{N}=5 \\
\text { (\% cases) }\end{array}$ & $\begin{array}{l}\text { Mixed Pf }+ \\
\mathrm{Pv} \mathrm{N}=9 \\
\text { (\% cases) }\end{array}$ & $\begin{array}{l}\text { Mean } \\
\text { placental } \\
\text { weight at } \\
\text { delivery/g (SD) }\end{array}$ & $\begin{array}{l}\text { Birth } \\
\text { weight/g } \\
\text { (SD) }\end{array}$ & Haematocrit (\%) \\
\hline CONTROLS N = 27 & $\begin{array}{l}\text { UK + Thai/Karen } \\
\text { controls }\end{array}$ & $29.6(6.2)$ & $38.2(2.42)$ & $2(0-5)$ & 0 & - & - & - & $493(127)$ & $\begin{array}{l}2951 \\
(457)^{b}\end{array}$ & $30.1(3.21)^{b}$ \\
\hline $\begin{array}{l}\text { TREATED MALARIA } \\
N=26\end{array}$ & $\begin{array}{l}\text { Malaria infection } \\
\text { during pregnancy } \\
\text { but not at delivery }\end{array}$ & $28.0(6.7)$ & $39.6(1.21)$ & $2(0-8)$ & $1(1-6)$ & 17 (65.4\%) & $3(11.5 \%)$ & $6(23.1 \%)$ & $524(130)$ & $\begin{array}{l}2923 \\
(322)\end{array}$ & $27.0(2.64)$ \\
\hline $\begin{array}{l}\text { ACTIVE MALARIA } \\
\mathrm{N}=14\end{array}$ & $\begin{array}{l}\text { Active placental } \\
\text { malaria at delivery }\end{array}$ & $24.7(6.5)$ & $39.1(1.59)$ & $0(0-4)$ & $1(1-7)$ & $9(64.3 \%)$ & $2(14.3 \%)$ & $3(21.4 \%)$ & $474(116)$ & $\begin{array}{l}2801 \\
(511)\end{array}$ & $27.2(3.13)$ \\
\hline $\begin{array}{l}\mathrm{p} \text { value across } \\
\text { groups }\end{array}$ & - & 0.084 & 0.09 & 0.048 & $0.83^{\mathrm{a}}$ & $1.0^{\mathrm{a}}$ & $1.0^{\mathrm{a}}$ & $1.0^{\mathrm{a}}$ & 0.56 & 0.692 & $0.0034^{*}$ \\
\hline
\end{tabular}

${ }^{a}$ Comparison does not include controls; ${ }^{b}$ No data from UK controls, $n=9 *$ significant. 
or Streck's fixative (PCA study), then subsequently embedded in wax and processed using standard histological techniques to produce paraffin blocks. All processing and histological staining was performed in batches at the same pathology laboratory. Four $\mu \mathrm{m}$ histological sections were cut on a rotary microtome, and stained with both haematoxylin and eosin and Giemsa for histological examination.

\section{Image analysis of placental villi}

The morphological study examined terminal, as opposed to stem or intermediate villi, because previous studies on the effects of disease on villous morphometry, and a pilot study in these cases, indicated that the predominant changes caused by disease are seen in these villi. Terminal villi were identified using a series of criteria including thinned trophoblastic lining, lack of muscularized arterioles, and more than $50 \%$ of the cross sectional area being occupied by vessels [8].

A total of 67 placenta specimens were used for image analysis by assessing four main parameters: the cross sectional surface area (size) of terminal villi; the number of capillaries per terminal villus; the number of terminal villi per high powered field (hpf); and the perimeter measurement of terminal villi, both the mean perimeter of individual villi and the total perimeter measurement in ten hpf.
Tissue sections were examined and digital images were captured from ten randomly selected $\times 400 \mathrm{hpf}$ per slide using an Olympus DP-20 colour video camera interfaced with an Olympus BX41 microscope. The images were scanned at a resolution of $1,600 \times 1,200$ pixels using Olympus DP-20 BSW software (Version 2.2) and stored. Only the intermediate zone of the placenta was used and areas where villi could not be distinguished, with placental infarction, intervillous fibrin deposition, or arterial vessels forming the primary stem and intermediate villi were avoided, as these can distort the observed villous size $[7,13]$. Only terminal villi showing outlines completely within the microscopic field were counted. Captured images were used as raw data to quantify the villous architecture using the image analysis software Image (Version 1.46, NIH, Maryland, USA) and to measure the area of terminal villi $\left(\mu \mathrm{m}^{2}\right)$, number of capillaries per terminal villous and perimeter of terminal villi $(\mu \mathrm{m})$.

\section{Area of the terminal villi $\left(\mu \mathrm{m}^{2}\right)$}

The images were first subjected to a preprocessing step (Figure 2) which involved the removal of non-relevant areas to leave only single terminal villi outlines, using an eraser tool in Photoshop (Version CS6, Adobe System Inc., California, USA) software. Images were then adjusted by ImageJ to convert them to greyscale 8-bit images. After adjusting the threshold, the area of terminal

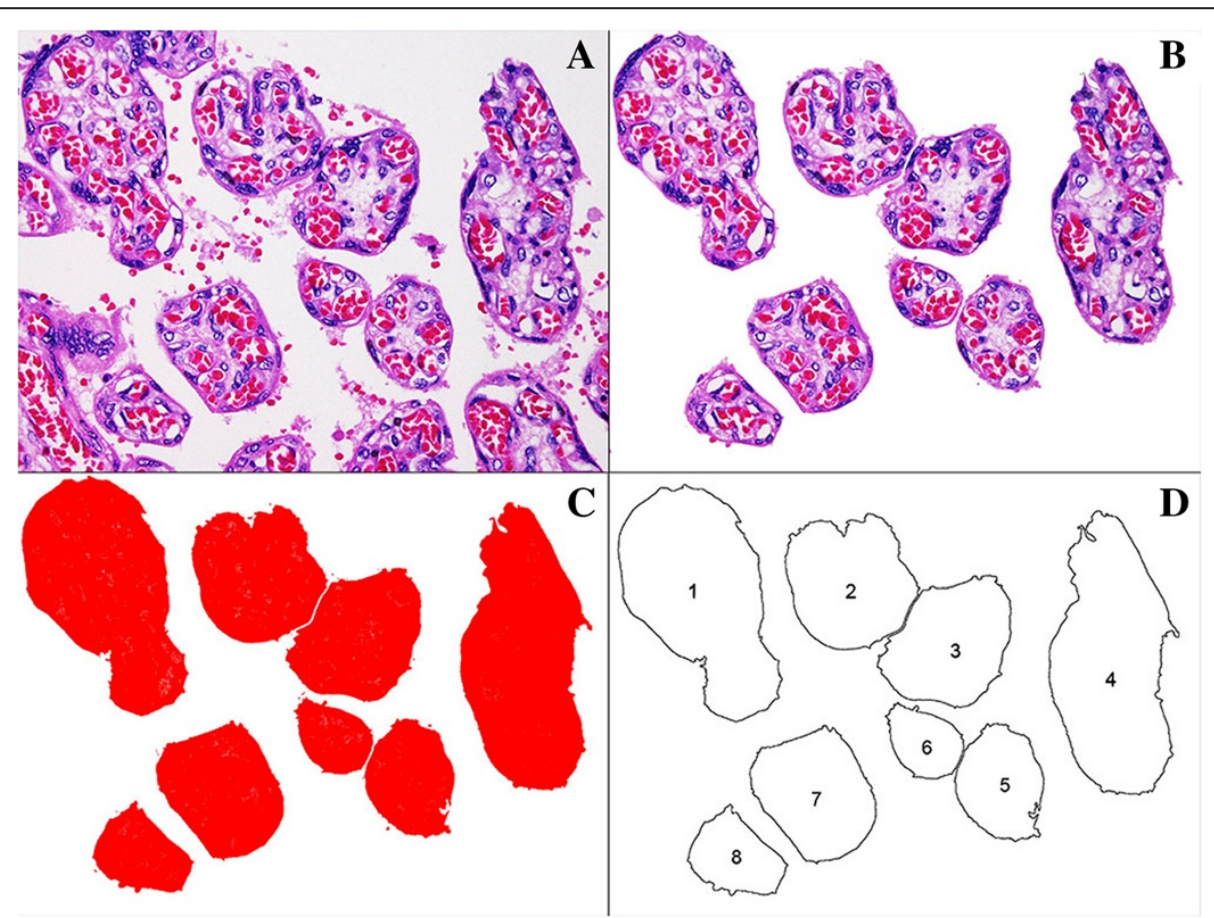

Figure 2 Image processing steps used to generate quantitative outputs from haematoxyling and eosin-stained placental sections. The various stages of processing are graphically illustrated in the following sequence: (A) original image (magnification $\times 400)$; (B) preprocessing step following removal of partial outlines and non-terminal villi; (C) adjusted threshold to generate solid red pixelated areas; (D) computerized measurement of area of terminal villi using the resultant outlines. 
villi was calculated as $\mu \mathrm{m}^{2}$ from the area of red pixels. The mean area of terminal villi for a case was calculated by the ratio between the total area and number of terminal villi in the field across all ten hpf.

\section{Number of capillaries per villous (villous vascularity)}

The villous vascularity was measured using the cell counter tool in ImageJ to count the number of open capillary spaces, lined by endothelium, in each villous (regardless of whether this contained erythrocytes or not). The number of capillaries per villous was calculated by the total number of villous vessels divided by the number of terminal villi across ten separate hpf.

\section{Number of terminal villi per high-powered field (hpf)}

The number of terminal villi in ten hpf was counted by eye with light microscopy in each case. The mean number of terminal villi per hpf in each patient was calculated by the ratio between the total number in ten hpf/ ten. Results for individual cases were then combined for comparison between clinical groups.

\section{Perimeter measurement of villi}

The perimeter of terminal villi (in $\mu \mathrm{m}$ ) was measured in two ways. The mean perimeter measurement of individual villous cross sections (for all villi in ten hpf) was calculated automatically in a similar manner to the villous area by ImageJ, directly from the outlined edge of terminal villi (Figure 2D). The total perimeter measurement of all villous cross sections in ten hpf was also calculated.

Histological scoring of changes in placental malaria infection The scoring system of Muehlenbachs et al. [22] was used to grade the presence or absence of parasitized red blood cells (PRBC), the density of inflammation and the presence of haemozoin pigment in intervillous fibrin. Semiquantitative scores for inflammation and pigment were also generated for each case (the histoscore). When present, infected erythrocytes were used to estimate a parasitaemia of infection in the placenta by counting infected and uninfected red blood cells over $100 \mathrm{hpf}$ and calculating the percentage of RBCs infected.

\section{Statistical analysis}

The specimens were prepared and analysed blind to the clinical group. Analyses were performed using the statistical program SPSS version 18 (SPSS Inc, Chicago, Illinois, USA) for Windows and STATA v12.0 (StataCorp LP, College Station, TX, USA). Data were described using means (standard deviation-SD) and frequencies (\%). Due to the relatively small sample size and unequal variances across groups, non-parametric tests were used for comparisons. Correlations between villous number, size and vascularity and between clinical groups, across all individuals and between clinical outcome measures, were assessed using Kendall's rank correlation coefficient (tau). The relationship between histoscore parameters and measures of villous architecture between clinical groups, subgroups of the PCA cases, and with clinical outcomes such as anaemia, was performed using the Fisher's exact test and the Kruskall-Wallis test. Differences were considered statistically significant for p-values less than 0.05 . However, as this was an exploratory study, numerous comparisons were made and exact p-values (to the third decimal place) are reported so that the reader may apply Bonferroni corrections, if preferred (where the statistically significant $\mathrm{p}$-value is calculated as $\alpha / \mathrm{n}$, where $\alpha=0.05$ and $\mathrm{n}=$ number of tests).

\section{Results}

Maternal malaria in southeast Asia differs from the African setting in that the disease is not endemic, there is a substantial proportion of $P$. vivax as well as $P$. falciparum infections, and also mixed infection with both organisms. In addition the patients included in this study were frequently tested for malaria and treated when this was detected, so active placental malaria at delivery is rare and the duration of undiagnosed malaria infections during gestation is minimal [17]. In malaria-infected cases, including both active and treated patients (a total of eighty-six infective episodes) the mean maternal parasitaemia on diagnosis was $21,308 / \mu \mathrm{l}(+/-$ SD 102,150).

\section{Correlation between villous area, vascularity and villous number}

There was a significant inverse correlation between villous area and villous numbers observed per hpf (Kendall's tau $=-0.3216 ; \mathrm{p}=0.0001$ ) either examining all cases or individual clinical groups (Figure 3A), because larger villi meant that less villi were seen per hpf at a given magnification. These two measurements were therefore covariate and not independent as an outcome measure of placental pathology. Conversely, there was a significant positive correlation between villous area and villous vascularity (Kendall's tau $=0.4799 ; \mathrm{p}<0.0001$ ) (Figure 3B).

\section{Fixation and geographical origin influence observed villous architecture}

Several other potentially confounding factors influenced the observed villous area. The first was the method of fixation. Non malaria-infected Thai/Karen cases fixed in formalin had a non-significantly lower mean villous area than cases fixed with Streck's solution $(p=0.0842)$ but significantly lower villous vascularity ( $p<0.0002)$. In addition, the UK control group had a significantly greater villous area $(\mathrm{p}<0.0004)$ and villous vascularity ( $\mathrm{p}<0.0087)$ than Thai/Karen control cases indicating a 

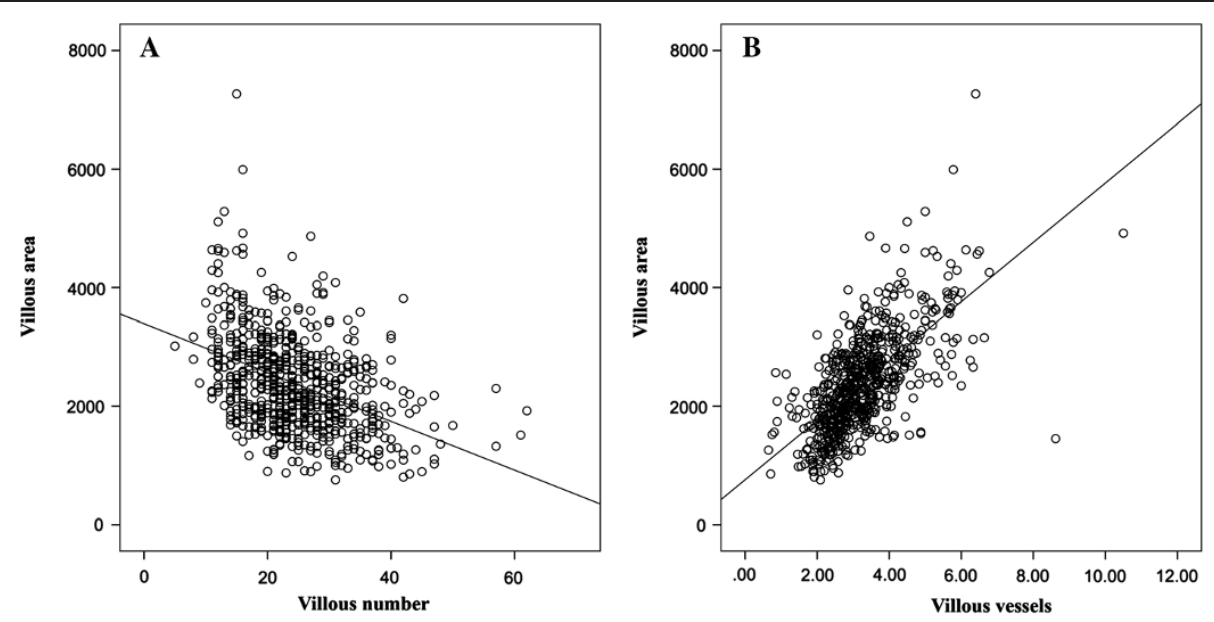

Figure 3 Correlations between the villous area, number of villi per high-powered field and villous vascularity. 3 A - Dot plot showing the negative correlation between the villous area and observed number of villi per hpf in individual cases (Line = line of best fit, Spearman rank correlation coefficient rho $=-0.425, p<0.0001$ ). $3 \mathbf{B}$ - Dot plot showing the positive correlation between the villous area and villous vascularity in individual cases (Line $=$ line of best fit, Spearman rho $=0.668, p<0.0001$ ).

difference in observed villous area on a geographical basis (Figure 4). Other placental villous architectural features, such as number of villi/hpf and perimeter measurements, were not significantly different between the two control groups. One potential explanation for this might be villous congestion in UK deliveries due to delayed clamping of the umbilical cord, which was not practised in Karen women at the time these samples were collected. UK control cases had higher body weights than Karen controls, but the placental weights at delivery between these populations was not significantly different.

A post-hoc analysis of the results comparing UK controls, UPS, PCA and active malaria groups separately confirmed the significant differences in villous morphology

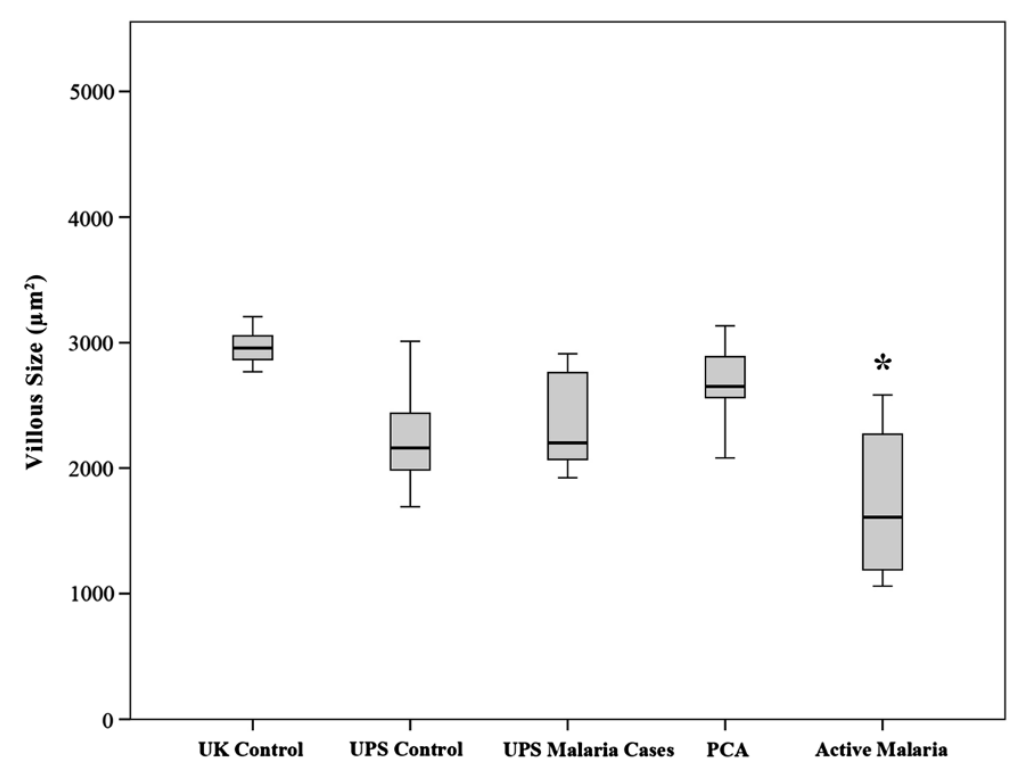

Figure 4 Boxplot graph comparing villous area between clinical groups of controls and cases derived from different fixative methods or geographical origin. A boxplot showing the distribution of villous area in sq $\mu \mathrm{m}$ between groups including UK controls, UPS cases (Thai/Karen cases fixed with formalin, divided into control non-malaria infected and malaria -infected UPS cases), PCA cases (Thai/Karen cases fixed with Streck fixative), and active placental malaria cases (Thai/Karen cases also fixed with formalin). UK controls showed significantly higher villous area than other groups ( $p<0.0004)$. PCA cases showed a non-significantly higher villous area than UPS controls fixed in formalin $(p<0.0842)$. Active placental malaria cases showed significantly lower villous areas than all other groups *. Box and whisker plots for this and subsequent figures include; line $=$ median, upper and lower bar $=25$ and $75^{\text {th }}$ centiles, respectively, with shaded box covering interquartile range; whiskers cover maximum and minimum values. 
across these groups, regardless of racial origin or fixation method. Therefore, despite the differences in villous architecture caused by the fixation method and geographical origin of the patient, outlined above, the analysis of the clinical groups compared combined control cases (UK and Thai/Karen controls; patients with no malaria infection) with all treated malaria cases (proven malaria infection at some point in gestation detected by screening and treated, but no observable PRBCs in the placenta at delivery) and active placental malaria cases (where histological evidence of parasites within the placenta confirmed acute malarial infection at delivery).

\section{Changes in villous architecture in malaria cases}

There was a significant decrease in villous area in active malaria-infected cases compared to both controls and treated malaria cases $(\mathrm{p}=0.0084)$ (Figure $5 \mathrm{~A}$, Table 2$)$. The number of capillaries per terminal villous (villous vascularity) in the malaria-infected patients was also significantly decreased compared to controls or treated malaria cases $(\mathrm{p}=0.0139)$ (Figure 5B, Table 2).

The number of villi seen per hpf was significantly increased in both in active and treated malaria cases compared to non-malaria controls ( $\mathrm{p}<0.0001$ ) (Figure 5C,
Table 2). The perimeter measurement of individual villi was significantly decreased in malaria cases compared to treated malaria cases or controls $(\mathrm{p}<0.0006$, Figure 5D, Table 2) but conversely the total perimeter measurement of all villi/10 hpf was significantly higher in both treated and active malaria groups than uninfected controls ( $\mathrm{p}<0.0004$, Figure 5E, Table 2).

Villous size and vascularity was reduced in acute placental malaria cases. The number of villi observed per hpf increased significantly in malaria cases even after treatment, and this effect lasted until delivery. However other changes such as the acute decrease in villous size and changes in vascularity, and the histological features of placental malaria, resolved given early detection and appropriate treatment. In addition, although these smaller villi in malaria cases showed a lower perimeter measurement, the total perimeter available for gas exchange per unit area was increased, possibly a compensatory mechanism.

These results are different to those reported by Crocker and colleagues [23], who showed similar villous numbers between non-infected placenta, malaria infected, past infected and active infected placenta, using the histopathological classification system of Bulmer et al. [24]. In the

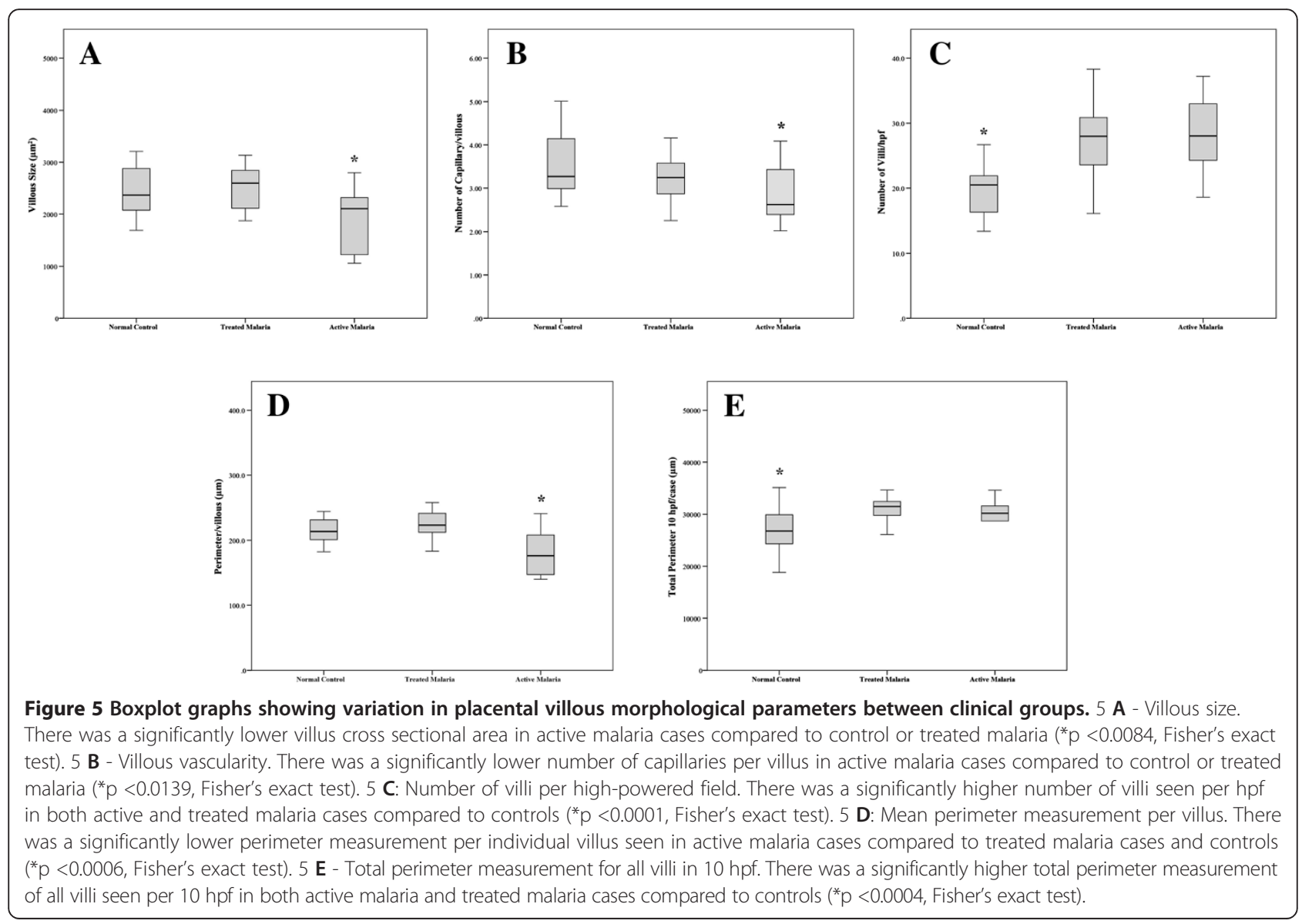


Table 2 Comparison of histoscore variables and measures of villous architecture between different clinical groups

\begin{tabular}{llll}
\hline Variable & $\begin{array}{l}\text { Controls vs Treated malaria } \\
\text { vs Active malaria }\end{array}$ & $\begin{array}{l}\text { P. falciparum vs } \\
\text { P. vivax or Mixed Pf/Pv }\end{array}$ & $\begin{array}{l}\text { Subgroups of PCA patients - } \\
\text { Treated Pf Early/Mid or late in gestation }\end{array}$ \\
\hline HISTOSCORE & & 1.000 & N/A (all -ve) \\
Presence of parasitized erythrocytes & $<0.001^{* *}$ & 0.177 & 0.382 \\
Inflammation & $<0.001^{* *}$ & 1.000 & 0.471 \\
Pigment & $<0.001^{* *}$ & 0.855 & $\mathrm{~N} / \mathrm{A}$ (all -ve) \\
Parasitaemia & $0.0001^{* *}$ & 0.346 & 0.481 \\
Inflammation score (I-III) & $0.001^{* *}$ & 0.613 & 0.335 \\
Pigment score (I-III) & $0.0002^{* *}$ & & \\
IMAGE ANALYSIS & & 0.321 & 0.554 \\
Villous area (mm $\left.{ }^{2}\right)$ & $0.0084^{* *}$ & 0.094 & 0.667 \\
Villous vascularity & $0.0139^{*}$ & 0.321 & 0.235 \\
Villi/hpf & $0.0001^{* *}$ & 0.629 & 0.203 \\
Perimeter/villous (mm) & $0.0006^{* *}$ & 0.119 & 0.523 \\
Total perimeter mm/10 hpf & $0.0004^{* *}$ & & \\
\hline
\end{tabular}

$\mathrm{Pf}=P$. falciparum, $\mathrm{Pv}=P$. vivax, ${ }^{*} \mathrm{P}<0.05,{ }^{*} \mathrm{P}<0.01 \mathrm{~N} / \mathrm{A}=$ Not Applicable.

patient groups examined in this study, significant changes in villous architecture were seen associated with active malaria infection, which resolved in treated malaria infection, apart from the observed number of villi/hpf.

\section{Histological scoring of placental pathological changes}

The pathological changes associated with placental malaria were scored according to the system of Muehlenbachs et al. [22]. The presence of parasites $(\mathrm{p}<0.001)$, degree of inflammation $(\mathrm{p}<0.001)$ and presence of parasite-derived haemozoin pigment $(\mathrm{p}<0.001)$ were all significantly different between active and treated malaria-infected cases. The inflammation score $(\mathrm{p}=0.001)$ and degree of parasitaemia in the placenta $(\mathrm{p}=0.0002)$ were also significantly associated with placental malaria infection (Table 2).

\section{Analysis of clinical outcomes and villous architecture}

There were no significant differences between the demographic details of the different clinical groups (Table 1) other than the maternal haematocrit, which was significantly higher in control cases than malaria treated or infected cases $(\mathrm{p}<0.0034)$. A number of other factors have been previously shown to influence villous size and architecture in other diseases, such as the gestational age of the placenta and placental weight at delivery. There was a small and (after correction for multiple comparisons) probably non-significant decrease in villous size associated with gestational age at delivery $(\mathrm{p}<0.043)$.

Malaria is the single greatest risk factor for anaemia in pregnant Karen patients and reversal is slow even when malaria and anaemia are treated $[19,25]$. Because anaemia might cause relative hypoxia within the placenta over a longer period, influencing villous size, the degree of anaemia in the mother (as measured by the haematocrit of the patients at the time of infection) was also correlated with the various measures of villous development above (area, number, vascularity and perimeter measurements). These results are shown in Table 3. There was a significant relationship between the degree of maternal anaemia at the time of infection (whether measured by absolute haematocrit or graded) and the subsequent number of observed villi at delivery, such that patients with moderate to severe anaemia at the time of infection had significantly more villi/hpf at delivery $(\mathrm{p}=0.0034)$ compared to uninfected controls (Figure 6). However no relationship was seen between villous size, vascularity and perimeter and the placental weight, maternal anaemia, gestational age or birth weight. The increase in villous numbers was also seen in treated malaria cases. These results suggest that changes to villous architecture may be dependent not only on malaria infection itself but on concomitant malariainduced anaemia, and persist despite effective treatment of the acute infection.

\section{Histology and placental villous architecture are similar in Plasmodium falciparum or Plasmodium vivax}

The relationship between villous architectural changes and the type of malaria infection was examined in malaria cases, for both monospecies infections with P. falciparum $(\mathrm{n}=26)$ or $P$. vivax $(\mathrm{n}=5)$, and where patients had both species either on separate occasions during gestation or mixed infection with both $(n=9)$. No significant differences were seen between any of the placental architectural parameters and either monoinfection with either $P$. falciparum or $P$. vivax, or in pregnancies where both species had been found, either as a mixed infection or on separate occasions (Table 2). The comparison of the morphological changes in the placenta 
Table 3 Correlation of villous architecture with clinical outcomes

\begin{tabular}{lccccc}
\hline Clinical parameter & Villous size/ $\mathbf{\mu m}^{\mathbf{2}}$ & $\begin{array}{c}\text { Vascularity } \\
\text { (number of vessels/villus) }\end{array}$ & $\begin{array}{c}\text { Number of } \\
\text { villi/10 hpf }\end{array}$ & $\begin{array}{c}\text { Villous perimeter } \\
(\boldsymbol{\mu m} / \mathbf{v i l l o s})\end{array}$ & $\begin{array}{c}\text { Villous perimeter } \\
\text { (total per 10 hpf) }\end{array}$ \\
\hline Placental weight & 0.995 & 0.547 & 0.975 & 0.761 & 0.208 \\
Gestational Age at delivery & $\mathbf{0 . 0 4 3 ^ { * }}$ & 0.364 & 0.247 & 0.968 & 0.310 \\
Maternal anaemia Grade $^{\mathrm{a}}$ & 0.656 & 0.354 & $\mathbf{0 . 0 0 3 4 ^ { * * }}$ & 0.525 & 0.214 \\
Maternal haematocrit \% & 0.142 & 0.922 & $\mathbf{0 . 0 0 9 *}$ & 0.760 & 0.346 \\
Birthweight & 0.833 & 0.567 & 0.441 & 0.454 & 0.179 \\
\hline
\end{tabular}

${ }^{a}$ Maternal anaemia graded by haematocrit at infection ( $0=$ normal, $1=25-30 \%, 2=<25 \%$, see Methods).

$\mathrm{b}_{\%}$ maternal haematocrit at infection.

Figures in bold are significant at ${ }^{*} \mathrm{P}<0.05$ and ${ }^{*} \mathrm{P}<0.01$.

in this study is however necessarily limited by the small number of samples from $P$. vivax patients, which could generate a bias in the analysis.

Histological descriptions of pure $P$. vivax placental malaria are so far limited [17,26-28]. Patients who had sequential (not simultaneous) $P$. falciparum and $P$. vivax infections occurring at different times in the same gestation appeared similar histologically, whichever infection was first, without differences in the degree of inflammatory response. Whether multiple recrudescent infections have clinically or pathologically different results from mono- or true dual infection remains to be determined.

\section{Relationship between timing of infection and delivery}

In PCA patients, all of whom had mono-infection with $P$. falciparum, selection was according to the timing of the last treated malaria infection compared to delivery. These groups were divided into 'early' ( $\mathrm{n}=6$ women who had a clinical episode of malaria treated at least five months prior to delivery and no subsequent infections); 'middle' ( $\mathrm{n}=6$ included those with malaria infection two to four months before delivery) and 'late' infection ( $\mathrm{n}=5$ those with treated malaria infection within one month before delivery). Comparison showed no significant differences between timing of infection before delivery and any of the parameters of the histoscore or villous architecture (Table 2), although the groups were small.

Because pathology at a given point in gestation can cause placental insufficiency, fibrinoid necrosis and scarring, subsequent development may be affected even if the infection is treated, and still be seen histologically at delivery some time later. However when groups of patients within the PCA study were compared, all of whom had been infected, diagnosed and treated for malaria but at varying times in gestation before delivery, there were no differences in villous architecture or resultant histoscore in the placenta at delivery. The similarity of both villous architecture and histoscore of treated placental

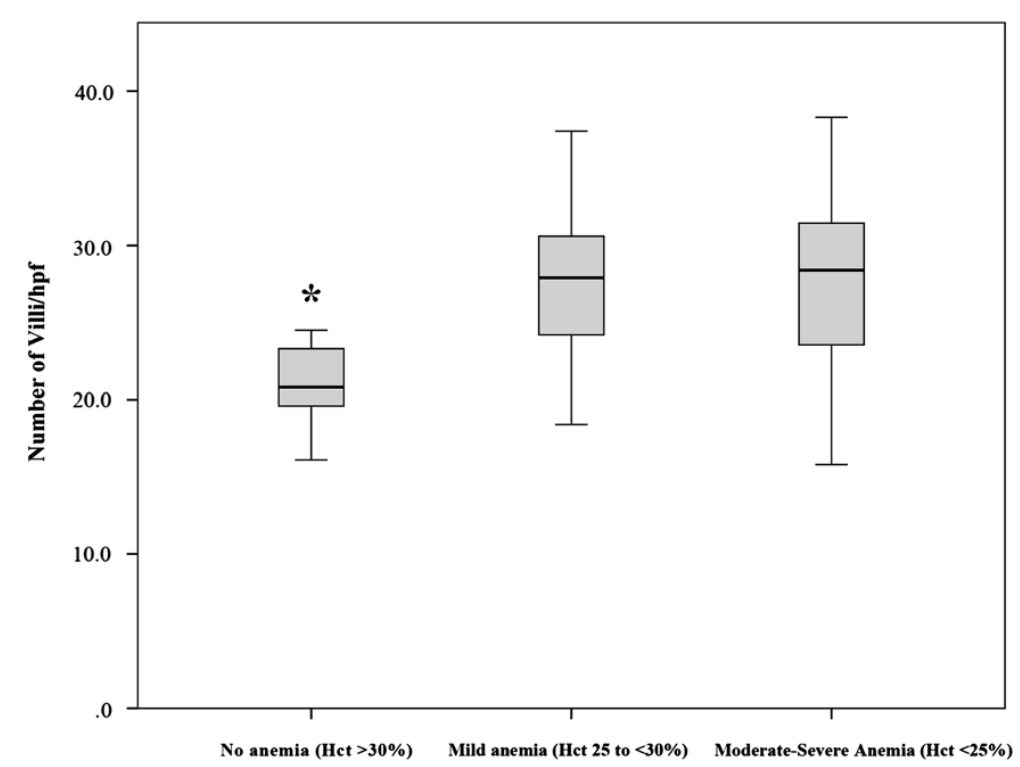

Figure 6 Boxplot graph of villous number per high-powered field compared to maternal anaemia at the time of infection. Boxplot showing the significantly higher number of villi seen per $10 \mathrm{hpf}$ in cases with mild and severe anaemia compared to patients without anaemia $\left({ }^{*} p<0.0034\right.$, Fisher's exact test). 
malaria with normal control levels implies that if malaria infection is detected and treated effectively, the placenta retains enough compensatory mechanisms to return to normal growth and development without long-term pathological changes caused by untreated malaria infection.

The acute reduction of villous size and perimeter associated with active malaria infection seems unlikely to reflect decreased blood flow in the placenta before delivery (for instance due to the effects of vascular obstruction by sequestered parasites, leukocytes or fibrin deposition) because these processes affect the maternal blood space. Changes in villous vascularity and size in other diseases examined using morphometry developed over a longer period due to poor oxygenation and hypertension, leading to defective stem villous development and hence vascularization of terminal villi $[16,29,30]$. Apart from blood flow, the activity of various growth-promoting hormones, such as insulin-like growth factor or placental growth factor, plays a role in supporting normal placental development [31], but changes in these growth axes would not explain the acute shrinkage seen in malaria infection. This study specifically examined changes in terminal villi only, and therefore excluded areas that may be affected by other pathological changes caused by placental malaria infection, such as infarction or extensive intervillous fibrin deposition. Such changes also have an important effect on placental function and the extent of these lesions is another important factor affecting the structure and function of the remaining placenta.

Villous size was interdependent with villous vascularity. If there was a generalized decrease in villous vessel numbers in malaria infection this may cause an acute shrinkage of villous area, which seems more likely than a sudden loss of established villous blood vessels. Placental malaria infection causes acute changes to both maternal and foetal haemodynamics, which reverses rapidly, within three days, after effective treatment [32,33]. Foetal heart rate is increased and it may be that other physiological parameters of the foetal-maternal circulation in the placenta, such as blood pressure, are altered in acute infection. Whether this reduces the perfusion pressure of the villi or causes changes to villous size, which are maintained and seen on subsequent histology, is unclear. Ultrasound Doppler investigation of the placenta during acute malaria also shows that malaria is associated with significantly smaller placental volumes, which can be linked to subsequent IUGR and poor outcome [34].

The pathophysiological effects of malaria infection in the placenta include inflammatory cell localization, cytokine release and direct effects on villous function by parasitized erythrocyte binding [35-38]. Cytokine and chemokine release, induced in host leukocytes and syncytiotrophoblastic cells and enhanced by phagocytosis of malaria pigment [39], causes auto-induction of chemokines and angiogenic factors which can attract host monocytes [40]. In other organs where PRBC and leukocytes are sequestered, signalling across endothelial monolayers causes changes to interendothelial cell junctions and oedema formation, such as in the brain during cerebral malaria [41]. There is evidence that adhesion of PRBC to syncytiotrophoblastic cells initiates signalling in these cells by tyrosine phosphorylation [42]. The current findings suggest another potential mechanism for placental dysfunction during malaria infection, where the presence of $\mathrm{PRBC}$ and host leukocytes reduces villous surface area, essential for foeto-maternal transfer of gas or nutrients, and disposal of potentially toxic metabolites, affecting foetal growth and development in undiagnosed or recurrent infections. This may result from either increased external osmotic pressure on the villus, or changes to foetal and maternal blood pressure reducing vascular filling, causing acute shrinkage. However the increase in relative villous numbers and therefore total available villous perimeter per unit area, which persists after treatment of infection through to delivery, hints at an attempt at compensation for this.

\section{Conclusions}

Digital image analysis was used to examine pathological changes to villous architecture in patients with active and treated malaria and controls. Villous area was significantly correlated with vascularity and inversely correlated with density of villi per unit area. UK controls showed significantly greater villous area and vascularity than Karen groups. There was a significant relationship between villous number and anaemia at the time of infection, but not placental weight and gestational age at delivery. Active malaria-infected cases showed significantly smaller villous area, vascularity, density, and perimeter measurement than controls. However increased villous numbers in malaria cases caused a compensatory increase in total available villous perimeter. Histology score and villous architecture showed no significant differences between infections with either $P$. falciparum, $P$. vivax, or consecutive infections by both during the course of pregnancy. These results imply that villous architecture changes acutely during malaria infection, causing shrinkage of the terminal villous and compensatory increase in the number of villi which persists following treatment and eradication of the infection. It cannot be excluded that the reversal of placental morphological changes may be related to improvement in maternal anaemia, caused by malarial treatment. However other architectural and pathological changes induced by malaria infection are reversible given early detection and appropriate anti-malarial treatment. 


\section{Competing interests}

The authors declare that they have no competing interests.

\section{Authors' contributions}

SC, AM, EP, and GDT performed the experiments, analysed data and wrote the paper. UC, EP, PV, RM, MJR, and FN collected samples, helped analyse data and wrote the paper. SC and SJL performed statistical analysis. GDT, UC, $E P, R M, M J R$, and FN conceived of the study and designed experiments. All authors read and approved the final manuscript.

\section{Acknowledgements}

We would like to thank the pregnant women who agreed to placental biopsy both at the Department of Tropical Pathology and at SMRU; and the midwives, laboratory technicians and members of the logistic departments who helped to collect and process the specimens, in particular our thanks to Lily Kiricharoen and Pi Poo for help with each of the SMRU studies. We thank Dr Sumate Ampawong for supervision and training for image analysis techniques. This study was supported by The Office of the Higher Education Commission and Mahidol University under the National Research Universities Initiative, the Faculty of Tropical Medicine and the Wellcome Trust of Great Britain, supported by grant reference 089275/Z/09/Z.

\section{Author details}

'Department of Tropical Pathology, Faculty of Tropical Medicine, Mahidol University, 420/6 Rajvithi Road, Bangkok 10400, Thailand. ' Mahidol-Oxford Research Unit, Faculty of Tropical Medicine, Mahidol University, 420/6 Rajvithi Road, Bangkok 10400, Thailand. 'Shoklo Malaria Research Unit, Mae Sot, Thailand. ${ }^{4}$ Department of Pathology, University of Washington, Seattle, WA 91895, USA. ${ }^{5}$ Centre for Tropical Medicine, Nuffield Department of Clinical Medicine, University of Oxford, Oxford OX3 7LJ, UK.

Received: 14 October 2013 Accepted: 28 December 2013

Published: 4 January 2014

\section{References}

1. WHO: Malaria in pregnancy: Guidelines for measuring key monitoring and evaluation indicators. Geneva: World Health Organization; 2007.

2. Nosten F, Rogerson SJ, Beeson JG, McGready R, Mutabingwa TK, Brabin B: Malaria in pregnancy and the endemicity spectrum: what can we learn? Trends Parasitol 2004, 20:425-432.

3. Rogerson SJ, Mwapasa V, Meshnick SR: Malaria in pregnancy: linking immunity and pathogenesis to prevention. Am J Trop Med Hyg 2007 77:14-22.

4. Desai M, ter Kuile FO, Nosten F, McGready R, Asamoa K, Brabin B, Newman $\mathrm{RD}$ : Epidemiology and burden of malaria in pregnancy. Lancet Infect Dis 2007, 7(2):93-104.

5. Bardaji A, Sigauque B, Sanz S, Maixenchs M, Ordi J, Aponte JJ, Mabunda S, Alonso PL, Menendez C: Impact of malaria at the end of pregnancy on infant mortality and morbidity. J Infect Dis 2011, 203:691-699.

6. Castellucci M, Kaufmann P: A three-dimensional study of the normal human placental villous core: II Stromal architecture. Placenta 1982, 3:269-285.

7. Thliveris JA, Baskett TF: Fine structure of the human placenta in prolonged pregnancy. Preliminary report. Gynecol Obstet Invest 1978, 9:40-48.

8. Huppertz B: The anatomy of the normal placenta. J Clin Pathol 2008, 61:1296-1302.

9. Walter PR, Garin Y, Blot P: Placental pathologic changes in malaria. A histologic and ultrastructural study. Am J Pathol 1982, 109:330-342.

10. Boyd PA, Scott A: Quantitative structural studies on human placentas associated with pre-eclampsia, essential hypertension and intrauterine growth retardation. Br J Obstet Gynaecol 1985, 92:714-721.

11. Calderon IM, Damasceno DC, Amorin RL, Costa RA, Brasil MA, Rudge MV: Morphometric study of placental villi and vessels in women with mild hyperglycemia or gestational or overt diabetes. Diabetes Res Clin Pract 2007, 78:65-71.

12. Giles WB, Trudinger BJ, Baird PJ: Fetal umbilical artery flow velocity waveforms and placental resistance: pathological correlation. Br J Obstet Gynaecol 1985, 92:31-38.
13. Egbor M, Ansari T, Morris N, Green CJ, Sibbons PD: Pre-eclampsia and fetal growth restriction: how morphometrically different is the placenta? Placenta 2006, 27:727-734.

14. Almasry SM, Elfayomy AK: Morphometric analysis of terminal villi and gross morphological changes in the placentae of term idiopathic intrauterine growth restriction. Tissue Cell 2012, 44:214-219.

15. Zigic Z, Markovic S, Grbesa D, Ramic S, Halilovic A: Quantitative research of capillaries in terminal villi of mature placentae. Bosn J Basic Med Sci 2010, 10:147-152.

16. Macara L, Kingdom JC, Kaufmann P, Kohnen G, Hair J, More IA, Lyall F, Greer IA: Structural analysis of placental terminal villi from growth-restricted pregnancies with abnormal umbilical artery Doppler waveforms. Placenta 1996, 17:37-48.

17. McGready R, Davison BB, Stepniewska K, Cho T, Shee H, Brickman A, Udomsangpetch R, Looaresuwan S, White NK, Meshnick SR, Nosten F: The effects of Plasmodium falciparum and $P$. vivax infections on placental histopathology in an area of low malaria transmission. Am J Trop Med Hyg 2004, 70:398-407.

18. Rijken MJ, Papageorghiou AT, Thiptharakun S, Kiricharoen S, Dwell SL, Wiladphaingern J, Pimanpanarak M, Kennedy SH, Nosten F, McGready R: Ultrasound evidence of early fetal growth restriction after maternal malaria infection. PLoS One 2012, 7:e31411.

19. Rijken MJ, de Wit MC, Mulder EJ, Kiricharoen S, Karunkonkowit N, Paw T, Visser GH, McGready R, Nosten FH, Pistorius LR: Effect of malaria in pregnancy on foetal cortical brain development: a longitudinal observational study. Malar J 2012, 11:222.

20. McGready R, Tan SO, Ashley EA, Pimanpanarak M, Viladpai-Nguen J, Phaiphun L, Wustefeld K, Barends M, Laochan N, Keereecharoen L, Lindegardh $N$, Singhasivanon P, White NJ, Nosten F: A randomised controlled trial of artemether-lumefantrine versus artesunate for uncomplicated Plasmodium falciparum treatment in pregnancy. PLoS Med 2008, 5:e253.

21. Piola P, Nabasumba C, Turyakira E, Dhorda M, Lindegardh N, Nyehangane D, Snounou G, Ashley EA, McGready R, Nosten F, Guerin PJ: Efficacy and safety of artemether-lumefantrine compared with quinine in pregnant women with uncomplicated Plasmodium falciparum malaria: an open-label, randomised, non-inferiority trial. Lancet Infect Dis 2010, 10:762-769.

22. Muehlenbachs A, Fried M, McGready R, Harrington WE, Mutabingwa TK, Nosten F, Duffy PE: A Novel histological grading scheme for placental malaria applied in areas of high and low malaria transmission. $J$ Infect Dis 2010, 202:1608-1616.

23. Crocker IP, Tanner OM, Myers JE, Bulmer JN, Walraven G, Baker PN: Syncytiotrophoblast degradation and the pathophysiology of the malaria-infected placenta. Placenta 2004, 25:273-282.

24. Bulmer JN, Rasheed FN, Francis N, Morrison L, Greenwood BM: Placental malaria. I. Pathological classification. Histopathol 1993, 22:211-218.

25. Luxemberger C, McGready R, Khan A, Morison L, Cho T, Chongsuphajaisiddhi T, White NJ, Nostern F: Effects of malaria during pregnancy on infant mortality in an area of low malaria transmission. Am J Epidemiol 2001, 154:459-465.

26. Mayor A, Bardaji A, Felger I, King CL, Cistero P, Dobano C, Stanisic DA, Siba P, Wahlgren M, del Portilli H, Mueller I, Menendez C, Ordi J, Rogerson S: Placental infection with Plasmodium vivax: a histopathological and molecular study. J Infect Dis 2012, 206:1904-1910.

27. Souza RM, Ataide R, Dombrowski JG, Ippolito V, Aitken EH, Valle SN, Alvare JM Epiphanio S, Marinho CR: Placental histopathological changes associated with Plasmodium vivax infection during pregnancy. PLoS Negl Trop Dis 2013, 7:e2071.

28. Carmona-Fonseca J, Arango E, Maestre A: Placental malaria in Colombia: histopathological findings in Plasmodium vivax and $P$. falciparum infections. Am J Trop Med Hyg 2013, 88:1093-1101.

29. Gilio DB, Miranda Correa RR, de Oliveira Guimaraes Souza C, Peres LC, Marques Salge AK, Cavellani CL, de Paula Antunes Teixeira V, da Cunha Castro Costa E: Analysis of placenta vascularization in patients with uterine altered artery Doppler flow velocity exams. J Obstet Gynaecol Res 2009, 35:648-653.

30. Huisman MA, Timmer B, Stegehuis J, Swart B, Aarnoudse JG, Erwich JH: Vascularization in first-trimester chorionic villi in complicated and uncomplicated pregnancies. Am J Obstet Gynecol 2010, 202(88):e1-e7.

31. Forbes K, Westwood M: Maternal growth factor regulation of human placental development and fetal growth. J Endocrinol 2010, 207:1-16. 
32. Rulisa $S$, Kaligirwa N, Agaba S, Karangayire P, Mens PF, de Vries PJ: Fetal and maternal hemodynamics in acute malaria during pregnancy. Int $J$ Gynaecol Obstet 2012, 119:66-69.

33. Griffin JB, Lokomba V, Landis SH, Thorp JM Jr, Herring AH, Tshefu AK Rogerson SJ, Meshnick SR: Plasmodium falciparum parasitaemia in the first half of pregnancy, uterine and umbilical artery blood flow, and foetal growth: a longitudinal Doppler ultrasound study. Malar J 2012, 11:319.

34. Rijken MJ, Moroski WE, Kiricharoen S, Karunkonkowit N, Stevenson G, Ohuma EO, Noble JA, Kennedy SH, McGready R, Papageorghiou AT, Nosten FH: Effect of malaria on placental volume measured using three-dimensional ultrasound: a pilot study. Malar J 2012, 11:5

35. Brabin BJ, Romagosa C, Abdelgalil S, Menendez C, Verhoeff FH, McGready R, Fletcher KA, Owens S, d'Alledandro U, Nosten F, Fischer PR, Ordi J: The sick placenta - the role of malaria. Placenta 2004, 25:359-378.

36. Beeson JG, Duffy PE: The immunology and pathogenesis of malaria during pregnancy. Curr Top Microbiol Immunol 2005, 297:187-227.

37. Mens PF, Bojtor EC, Schallig HD: Molecular interactions in the placenta during malaria infection. Eur J Obstet Gynecol Reprod Biol 2010, 152:126-132.

38. Umbers AJ, Stanisic DA, Ome M, Wangnapi R, Haneih S, Unger HW, Robinson LJ, Lufele E, Biawog F, Siba PM, King CL, Beeson JG, Mueller I, Rogerson SJ: Does malaria affect placental development? Evidence from in vitro models. PLoS One 2013, 8:e55269.

39. Lucchi NW, Sarr D, Owino SO, Mwalimu SM, Peterson DS, Moore JM: Natural hemozoin stimulates syncytiotrophoblast to secrete chemokines and recruit peripheral blood mononuclear cells. Placenta 2011, 32:579-585.

40. Abrams ET, Brown H, Chensue SW, Turner GD, Tadesse E, Lema VM, Molyneux ME, Rochford R, Meshnick SR, Rogerson SJ: Host response to malaria during pregnancy: placental monocyte recruitment is associated with elevated beta chemokine expression. J Immunol 2003, 170:2759-2764.

41. Medana IM, Turner GD: Human cerebral malaria and the blood-brain barrier. Int J Parasitol 2006, 36:555-568.

42. Lucchi NW, Koopman R, Peterson DS, Moore JM: Plasmodium falciparuminfected red blood cells selected for binding to cultured syncytiotrophoblast bind to chondroitin sulfate $A$ and induce tyrosine phosphorylation in the syncytiotrophoblast. Placenta 2006, 27:384-394.

doi:10.1186/1475-2875-13-4

Cite this article as: Chaikitgosiyakul et al: A morphometric and histological study of placental malaria shows significant changes to villous architecture in both Plasmodium falciparum and Plasmodium vivax infection. Malaria Journal 2014 13:4.

\section{Submit your next manuscript to BioMed Central and take full advantage of:}

- Convenient online submission

- Thorough peer review

- No space constraints or color figure charges

- Immediate publication on acceptance

- Inclusion in PubMed, CAS, Scopus and Google Scholar

- Research which is freely available for redistribution 